\title{
A novel dual-mode tracking device for on-line dose monitoring in hadron therapy
}

\author{
Cecilia Voena ${ }^{* \dagger}$ \\ Istituto Nazionale di Fisica Nucleare Roma and La Sapienza Università di Roma \\ E-mail: cecilia.voena@romal.infn.it
}

\begin{abstract}
Hadron therapy is a technique for cancer treatment that exploits ion beams (mostly protons and carbons). Due to the shape of the hadron energy deposition pattern, dose releases are more localized with respect to radiotherapy. Hadron therapy is thus particularly suitable to treat tumors close to critical organs. A critical issue is the monitoring accuracy of the dose released by the beam to the patient. We present the design of a dual-tracking device capable of on-line dose monitoring through the detection of prompt photons and charged particles produced by the interactions of the beam in the patient tissues whose emission shapes are correlated to spatial dose release and to the Bragg peak. The dosimeter, whose design has been optimized using Monte Carlo simulations, is composed of a tracker made of six scintillating fiber stations followed by a layer of plastic scintillator (electron shield) and a lyso pixellated crystal to detect photons. A first tracker layer has been assembled and is under test. A complete simulation and reconstruction software has been developed to estimate the achievable spatial resolution. Charged particles are reconstructed using the fiber planes and those identified as protons are back-traced to determine the point of origin. Prompt photons are reconstructed exploiting their Compton interactions by combining the spatial and energy measurements from the tracker for the electron and from the lyso for the photon. For a real hadrotherapeutic treatment the achievable resolution is of the order of ten (few) millimeters using the neutral (charged) component.
\end{abstract}

53rd International Winter Meeting on Nuclear Physics,

26-30 January 2015

Bormio, Italy

*Speaker.

$\dagger$ on behalf of the ARPG group 


\section{Tumor treatment with radiation}

Proton and carbon ion beams are presently used to treat many different solid cancers. Compared to the standard X-rays treatments (radiotherapy) the main advantage of this technique is the better localization of the irradiation dose in the tumor region that allows to spare healthy tissues and possible surrounding organs. This because heavy charged particles loose most of the energy at the end of their range, in the Bragg peak, while X-rays exponentially decrease their energy with the penetration in matter. Moreover, hadrons have great efficiency in killing tumor cells and thus are more effective in curing radioresistent tumors; on the other hand they need sophisticated facilities. By combing few hadron beams is possible to cover a given tumor volume (with the so called Spread Out Bragg Peak) sparing healthy tissues. Up to now most patients have been treated with proton beams, but use of carbon beams has now started. Protons, which are lighter than carbons, suffer more multiple Coulomb scattering that spreads the dose in the patient tissues but have reduced fragmentation at the end of the pattern. Carbon beams are more effective in hypoxic tumors.

\section{Dose monitoring in hadron therapy}

Together with improving precision of hadron therapy in tumor irradiation comes the necessity of new, more precise, monitoring techniques. The treatments are extremely sensitive to anatomical changes and patient mis-positioning, with related errors in the dose release (under-dosage to tumor cells, over-dosage to healthy tissues). Since the beam is completely absorbed in the patient, transmitted beam cannot be used for monitoring purposes, as opposed to radiotherapy. Currently the dose release is measured using beam monitor information or with PET (Positron Emission Tomography) scans just after the treatment. Promising new monitor techniques exploit the flux of secondary particles created by the beam interaction in the patient, namely: photons from $\beta^{+}$ emitters [1], prompt photons within the 1-10 MeV range [2] and charged particles [3], [4]. The detector should provide the dose measurement on-line, respecting the stringent space constraints in the treatment rooms.

\subsection{Photons from $\beta^{+}$emitters}

Collinear $511 \mathrm{keV}$ photons are produced by annihilation from $\beta^{+}$emitters created by the beam (e.g. ${ }^{11} C,{ }^{13} N$ ); it has been shown that the emission shape is correlated with the dose deposition for both proton and carbon ion beams as shown in Fig. 1 a) from a simulation based on the package FLUKA. In principle the PET technique can be used to detect the two photons but the spatial constraints in the treatment room prevent the use of a standard PET detector. Offline PET can be used, but metabolic wash-out during patient movement after the treatment deteriorates the achievable resolution while in-beam solutions are currently under study.

\subsection{Prompt photons}

The correlation of the prompt photons emission shape with the beam path is known in literature both for proton and carbon beam [5, 2]. The advantage of prompt photons is that they are produced more abundantly than other secondaries but on the other hand a huge neutron background is present. Moreover, it is not easy to back-point the photon direction, usual SPECT techniques are not well suited given the energy range (1-10 MeV). 

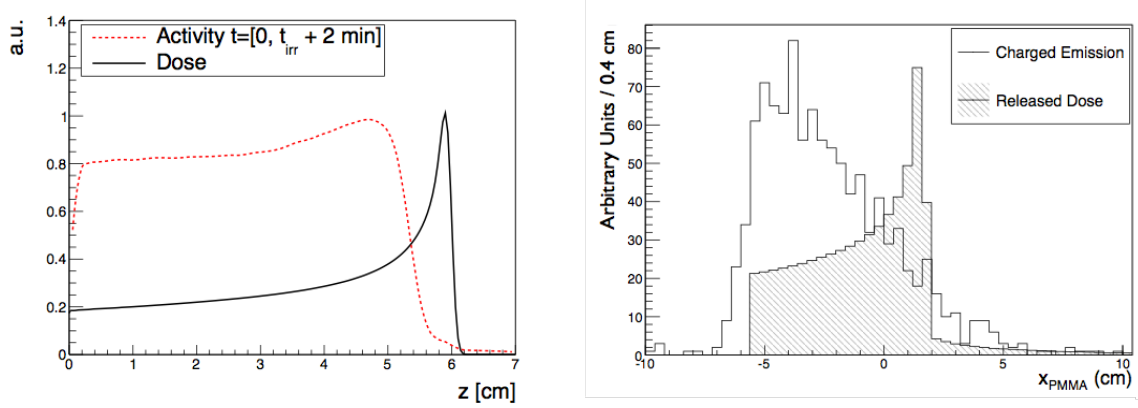

Figure 1: a) FLUKA MC simulation comparison between dose distribution (solid line) superimposed to the longitudinal profile of $\beta^{+}$activity for $95 \mathrm{MeV}$ proton beam on PMMA phantom. b) FLUKA simulated depth-dose distribution (hatched) superimposed on the longitudinal profile (solid line) of charged secondary particles as a function of the position on a PMMA phantom irradiated by a ${ }^{12} \mathrm{C}$ beam.

\subsection{Charged particles}

The flux of charged particles is lower with respect to that of prompt photons but the detection efficiency is very high and the original direction can easily back-tracked. Recent measurement of fluxes at different energies and angle are available in $[3,4]$ and it has been shown that the Bragg peak position can be linked to the charged particles emission as shown in Fig. 1 b).

\section{The dose profiler}

The two-fold nature of a single detector will allow to maximize the information on dose deposition that can be collected during a conventional treatment, considering its highly restrictive time constraints. Lack of statistics, together with space limitations are the most challenging issues to overcome. The requirements taken into account in the detector design are compactness, reliability, large geometrical acceptance, high tracking efficiency. The dose profiler is part of the INSIDE (Innovative Solutions for In-beam Dosimetry in hadron herapy) project born from the collaboration of a number of italian universities and INFN. The complete detector foresees also a detector for photons from $\beta^{+}$decays, made of 2 planar PET heads that is not described here. It will be installed in the CNAO treatment room with the layout shown in Fig. 2 a). The mechanical structure of the profiler is shown in Fig. 2 b). The tracker is composed by six planes each made by two orthogonally placed scintillating fiber layers (384 fibers each) to provide bi-dimensional view. Dimensions, spacing, thickness and materials have been optimized using Monte Carlo simulations. The scintillating fibers are $0.5 \times 0.5 \mathrm{~mm}^{2}$ with the minimal plane separation $(2 \mathrm{~cm})$ allowed by fibers front-end electronics readout, in order to increase the geometrical acceptance and the compactness of the detector. The readout is performed by means of $1 \times 1 \mathrm{~mm}^{2}$ silicon photomultipliers (SiPM), each coupled with two adjacent fibers. In total the $19.2 \times 19.2 \mathrm{~cm}^{2}$ sensitive area is read by 192 channels per layer. The spatial single hit resolution is $\sim 300 \mu \mathrm{m}$ and a rough hit energy information is available with $\sim 30 \%$ resolution for a minimum ionizing particle. A first layer of the tracker has been assembled and is currently under test. A plastic scintillator has been placed just after the tracker, in order to prevent any recoil Compton electron from reaching the calorimeter thus deteriorating pattern recognition. It is a polyvinyltoluene based scintillator and due to its 

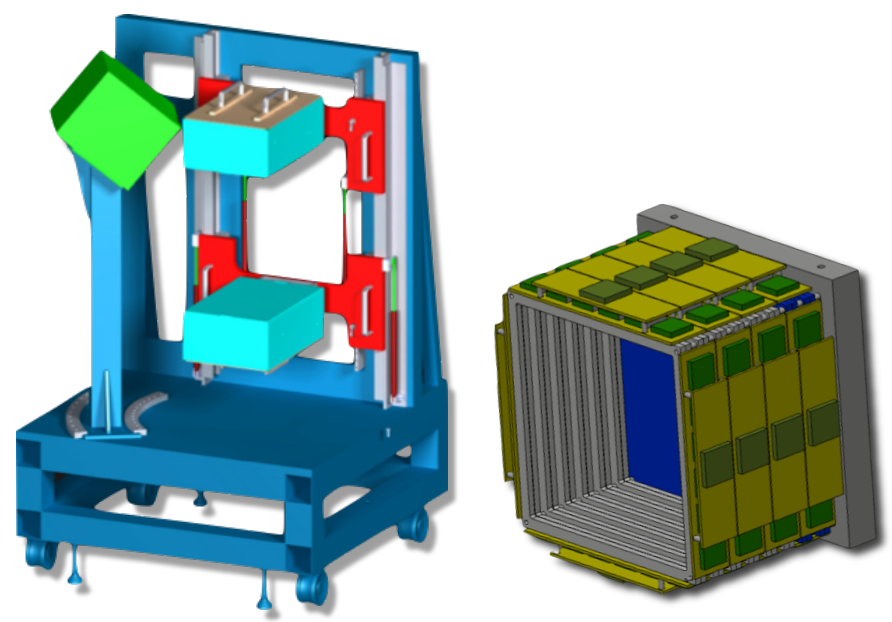

Figure 2: a) The cart design for the CNAO treatment room: in light blue the two PET-heads with their movements (red), in green the Dose Profiler box (60 degrees from the beam line) and in blu the mechanical structure. b). Sketch of the dose profiler mechanical structure.

low atomic number $\left(\mathrm{Z}_{e f f}=3.4\right)$ drastically reduces the amount of back-scattering of electrons. To avoid the development of a dedicated readout system, the front-end board of the tracker is also used to read the scintillator.

The high density compact scintillator placed behind the tracker is mainly used for Compton photon direction reconstruction. It consists of a $64 \times 64$ matrix of pixelated lyso crystals arranged in $4 \times 4$ blocks and, with its high atomic number $\left(Z_{e f f}=66\right)$, allows a compact design together with a high energy resolution, about $7 \%$ in the energy range of interest. The pixel layout allows to insulate photon position in the transverse plane at the cost of loosing the depth of interaction reconstruction capability. The crystal readout is performed by means of Multi Anode Photo-Multipliers.

\section{Achievable spatial resolution}

A detailed simulation of the detector based on FLUKA has been developed in order to optimize the detector design. Reconstruction software has been developed in $\mathrm{C} / \mathrm{C}++$ and has been directly interfaced with the analysis framework ROOT. For prompt photons, the probability of a Compton interaction inside a $500 \mu \mathrm{m}$ of scintillating fiber is rather low (of the order of $2 \%$ ). The probability of having a Compton interaction with at least three scintillating planes hit and the scattered photon in the lyso crystal drops this probability to $0.1 \%$ in the energy range of interest. Pair conversions of prompt photons occur in a small fraction of the case and are not considered in the reconstruction presently. Protons interacts in all the crossed fiber planes. Charged tracks are reconstructed with a track finding algorithm that starts from deposits in the fibers grouped together to form 3-dimensional clusters. Photons are identified from the light pattern in the lyso crystal. Prompt photons are reconstructed through their Compton interactions by reconstructing the scattered photon and electron. Prompt photon and proton events are distinguished on the basis of the energy deposit in the scintillators. 

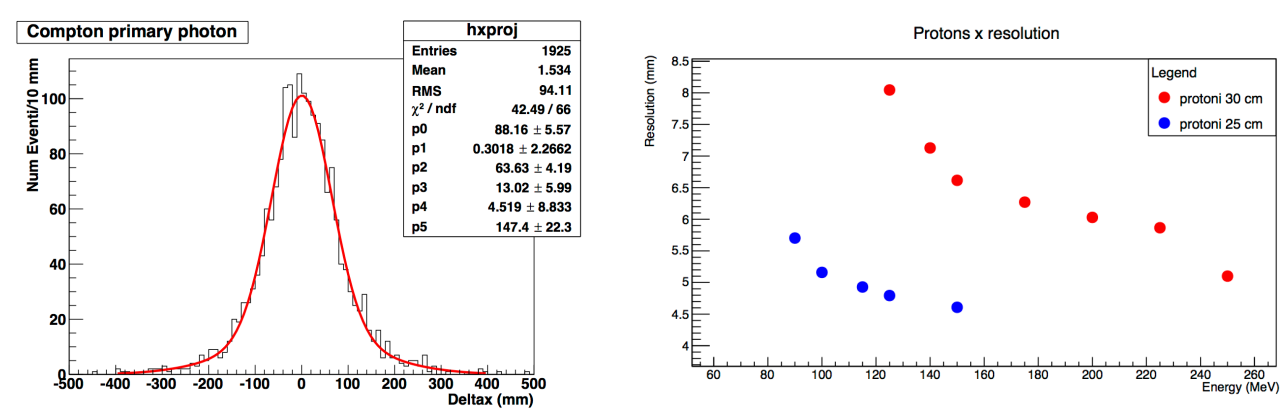

Figure 3: a) Distribution of the difference between the reconstructed and the true emission position of prompt photons reconstructed with the dose profiler estimated with the FLUKA Monte Carlo simulation described in the text. A two-Gaussian fit is superimposed. b) Spatial resolution for protons reconstructed with the dose profiler for different energies and depths of the proton source. (FLUKA Monte Carlo simulations described in the text).

The performances in terms of spatial resolution on the emission point of prompt photons have been evaluated with a FLUKA Monte Carlo simulation where a point-like source emits photons in the profiler acceptance with the expected energy spectrum for a $80 \mathrm{MeV} / \mathrm{u}$ carbon beam. Figure 3 a) shows the distribution of the difference between the reconstructed and the true photon origin. The width of the distribution $(9 \mathrm{~cm})$ is the resolution achievable with a single photon measurement, which becomes about $8 \mathrm{~mm}$ considering the prompt photons from an irradiated tumor slice in a realistic hadrotherapeutical treatment.

In order to measure the performances using the charged particles escaping the patient, a simulation with a patient head, schematized by two concentric spheres made of cortical bone and brain materials, has been considered. Two proton sources have been placed in the center of the sphere and at $5 \mathrm{~cm}$ from the center in order to reproduce two clinical scenarios: a worst case where the tumor is located exactly in the center of the patient head, and an intermediate case where the tumor is closer to the patient bone. Proton's inital kinetic energy has been variated between 75 and 250 $\mathrm{MeV}$ for the centered source and between $70 \mathrm{MeV}$ and $140 \mathrm{MeV}$ for the displaced source. The results for the spatial resolution obtained have been reported in Fig. 3 b); it converges at about 4.5 $\mathrm{mm}$ for single proton measurements, corresponding to $0.5 \mathrm{~mm}$ on an irradiated tumor slice in a realistic hadrotherapeutical treatment.

\section{Conclusion}

A new dose profiler to be used for on-line dose monitoring in hadron therapy is presented here. The principle of the detector is to reconstruct prompt photons and charged particles produced by the interaction of the beam and the tissues that escape the patient body. The particles emission pattern can be linked to the dose distribution. Promising results are obtained in terms of resolution from Monte Carlo simulations. The detector is currently under construction and the installation at CNAO (Pavia) is foreseen by the end of 2015 . 


\section{References}

[1] C. Agodi et al., "Study of the time and space distribution of $\beta^{+}$emitters from $80 \mathrm{MeV} / \mathrm{u}$ carbon ion beam irradiation on PMMA", Nucl. Inst., Meth. in Phys. Res. Section B, Beam Interaction with Materials, Atoms, vol. 283 n. 0, 2012.

[2] C. Agodi et al., "Precise measurement of prompt photon emission for carbon ion therapy", JINST 7 (2012) P03001. F. Bellini et al. "Extended calibration range for prompt photon emission in ion beam irradiation", Nucl.Instrum.Meth. A745 (2014) 114-118.

[3] C. Agodi et al., "Charged particle's flux measurement from PMMA irradiated by $80 \mathrm{MeV} / \mathrm{u}$ carbon ion beam", Phys. Med. Biol. 57 (2012) 5667.

[4] L. Piersanti et al., "Measurement of charged particles yields from PMMA irradiated by $220 \mathrm{MeV} / \mathrm{u}$ ${ }^{12}$ C, Phys. Med. Biol. 59 (2014) 1857-1872.

[5] E. Testa et al., "Dose profile monitoring with carbon ions by means of prompt-gamma measurements", Nucl. Instrum. Meth. B 267 (2009), 993. 